Document downloaded from:

http://hdl.handle.net/10251/103129

This paper must be cited as:

Sánchez Pérez, EA. (2017). Maharam-type kernel representation for operators with a trigonometric domination. Aequationes Mathematicae. 91(6):1073-1091. doi:10.1007/s00010-017-0507-6

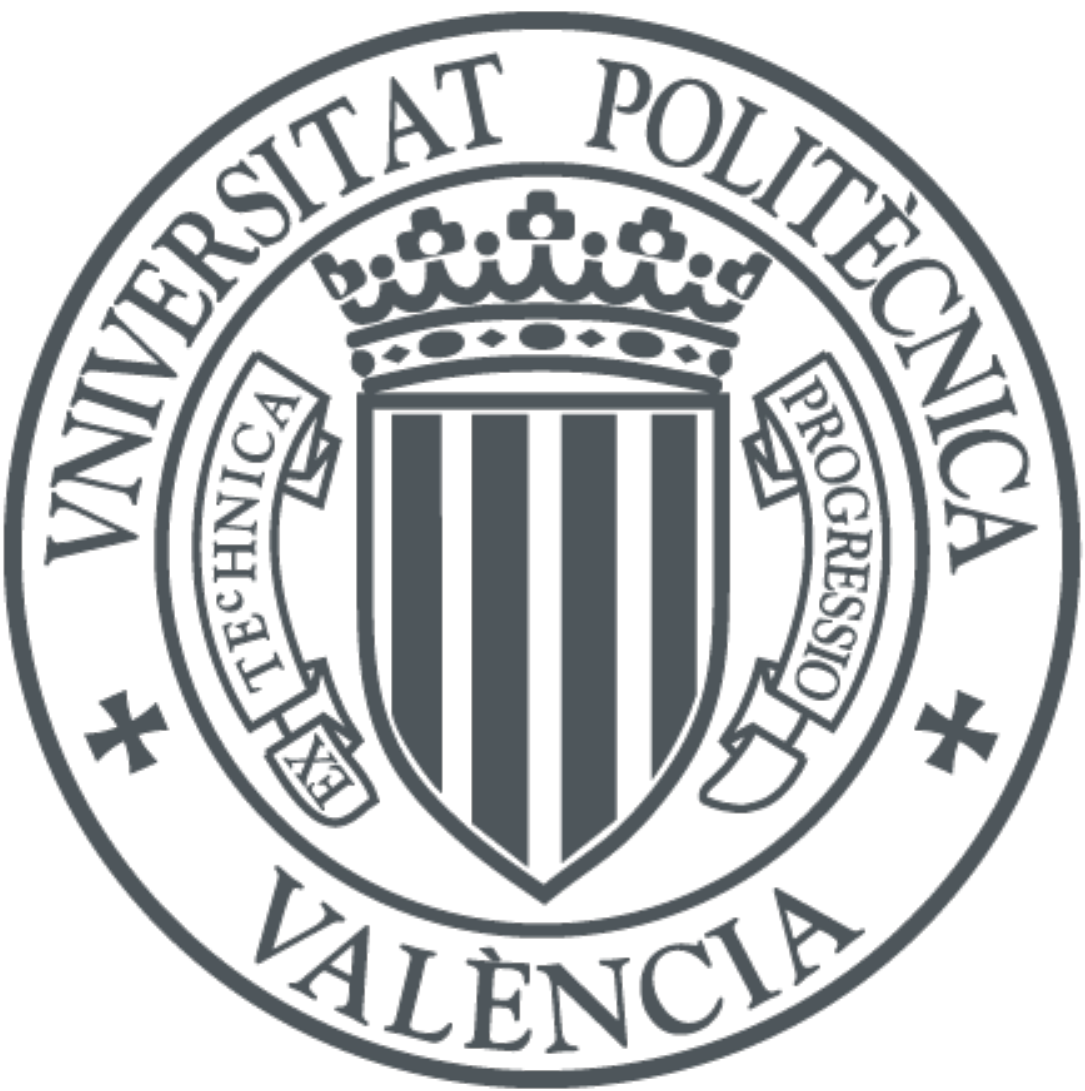

The final publication is available at

http://dx.doi.org/10.1007/s00010-017-0507-6

Copyright Springer-Verlag

Additional Information 


\title{
Maharam-type kernel representation for operators with a trigonometric domination
}

\author{
E. A. Sánchez Pérez
}

Mathematics Subject Classification (2010). Primary 42A10, 47B38; Secondary 42A24, 46E30, 46B28.

Keywords. Maharam Representation, Operator, Trigonometric Series, Domination, Hilbert Spaces.

\begin{abstract}
Consider a linear and continuous operator $T$ between Banach function spaces. We prove that under certain requirements an integral inequality for $T$ is equivalent to a factorization of $T$ through a specific kernel operator: in other words, the operator $T$ has what we call a Maharam-type kernel representation. In the case that the inequality provides a domination involving trigonometric functions, a special factorization through the Fourier operator is given. We apply this result to study the problem that motivates the paper: the approximation of functions in $L^{2}[0,1]$ by means of trigonometric series whose Fourier coefficients are given by weighted trigonometric integrals.
\end{abstract}

\section{Introduction}

Consider the (normalized) trigonometric system $\mathcal{T}=\left\{t_{k}: k \in \mathbb{N}\right\}$ in $L^{2}[0,1]$. In this paper we are interested in studying operators that are defined as the classical Fourier operator $\mathcal{F}: L^{2}[0,1] \rightarrow \ell^{2}$, but for which the associated Fourier-type coefficients $\beta_{k}$ are given by

$$
\beta_{k}(f)=\int_{[0,1]} f(w) t_{k}(w) h_{k}(w) d \mu, \quad f \in L^{2}[0,1],
$$

where $h_{k}$ are suitable bounded weights. Our motivation comes in part from the following classical problem. Depending on the function $f$ of $L^{2}[0,1]$ that is considered, the calculus of the integrals providing the genuine Fourier coefficients can have a big computational cost and the convergence of the associated Fourier series can be slow. To add some weights to the integrals appearing can of course solve the problem, but the orthogonality properties of the system $\mathcal{T}$ disappear when this procedure is used. This fact forces to use

This research has been supported by MTM2016-77054-C2-1-P (Ministerio de Economía, Industria y Competitividad, Spain). 
a different non-geometric approach. An abstract understanding of this well-known basic fact leads us to be interested in the functional analytic problem of describing the class of operators given by this procedure.

Therefore, the aim of the paper is to characterize the class of kernel-type operators between Banach function spaces that satisfy certain integral inequalities involving trigonometric series. Of course, the problem is not new. In fact, one of the classical topics of the functional analysis is to find good characterizations of integral operators between spaces of integrable functions by means of domination relations. Although there is a big amount of classical relevant papers in the topic, we have chosen two concrete ideas to provide an appropriate introduction for our results. The first one is given by the paper [16] of D. Maharam, in which an order domination between what she called $F$-integrals leads to write one of them as a kernel operator with respect to other: in her words, one of them has a kernel representation in terms the other one (see the Introduction, Theorem 7 and the previous Lemma in [16]; see also [15]). In these papers by Maharam the reader can find a reference to a representation theorem appearing in the paper [10] by $\mathrm{L}$. Kantorovitch and B. Vulich. Previous works by S. Bochner, G. Birkhoff and N. Dunford directly related to this topic are quoted in this paper.

The general framework in which these integral representation theorems were proved is given by what we may identify as a vector measure approach nowadays. These ideas were also used to find the maximal domain of the operators represented by these integrals (see Section 5 in [16]). The reader can find recent similar developments in [19] and [4, S.3 and S.4]. In the last mentioned paper, a generalized Radon-Nikodým Theorem is used in order to give conditions that allow to write an operator in terms of another given one using scalar domination between vector measures. This argument will be used in this paper.

The second idea we want to bring here is the well-known characterization of the Carleman operators (integral operators) in $L^{2}$-spaces. Theorem 17.2 in [9] states that an operator $A$ in $L^{2}$ is a Carleman operator if and only if there is a measurable function $h$ providing a pointwise domination for $A$ as

$$
|A(x)| \leq\|x\|_{L^{2}} \cdot|h|, \quad x \in L^{2} .
$$

Moreover, $h$ belongs to $L^{2}$ if and only if $A$ is a Hilbert-Schmidt operator ([9, Cor.17.3]). In the same chapter of this book the reader can find the following question: Is it possible to characterize the classes of integral operators by means of such kind of domination properties? ([9, Problem 17.4]).

In this direction, the aim of this paper is to provide a general procedure for characterizing specific classes of integral operators having some fixed type of kernel, with some integral domination inequalities among operators. Thus, we will show first some results for operators acting in Banach function spaces in which some integral inequalities involving two operators imply the factorization of the first one through a kernel operator that is constructed using the second one. This will be done in Section 3, where some rather technical procedures based on the Fremlin lattice tensor product of spaces of multiplication operators are used. Besides of the classical paper [7] of D.H. Fremlin, the main elements that are needed can be found in the paper [22] by A.R. Schep. The factorization is then obtained by a new version of the fundamental Hahn-Banach separation argument that is always used for proving such results, which is adapted for the 
tensor product setting from the one explained in [4]. After showing some applications to the case of averaging operators, we will use our technique for providing in Section 4 the integral domination inequalities that characterize the class of weighted Fourier-type operators mentioned above. Weighted norm inequalities for Fourier multipliers operators is a classical and current interesting topic; our results would find applications using such kind of domination, for the linear case and also for the multilinear case (see for example [8] and the references therein).

Another example of domination between pairs of operators that has been recently studied - in this case, pointwise domination - can be found in [6] and related references. In this case, the aim is to analyze which properties of the operator $T$ are inherited by the operator $S$ if the relation $|S| \leq|T|$ holds. Our techniques are different, although our abstract construction would also find applications in this setting.

\section{Basic concepts and notation}

Let us present first some special notations and concepts that will be used in the paper. If $E$ is a Banach space, we write $E^{*}$ for its dual and $B_{E}$ for its unit ball. We give the general reference [14] for concepts and results regarding Banach lattices and Banach function spaces. If $s$ is a sequence and $j \in \mathbb{N}$, we write as usual $s_{j}$ for the $j$-th coordinate; if $J \subseteq \mathbb{N}$, we will write $s_{J}$ for the sequence defined as $s_{j}$ for the $j$-th coordinate if $j \in J$, and 0 otherwise. Let $(\Omega, \Sigma, \nu)$ be a $\sigma$-finite measure space. Let $L^{0}(\mu)$ be the space of all ( $\mu$-a.e. equal equivalence classes of) measurable real functions on $\Omega$. $L^{\infty}(\mu)$ will denote the space of functions in $L^{0}(\mu)$ which are bounded $\mu$-a.e. By a Banach function space we mean a Banach lattice $X(\mu) \subset L^{0}(\mu)$ with norm $\|\cdot\|_{X}$ such that if $f \in L^{0}(\mu), g \in X$ and $|f| \leq|g| \mu$-a.e. then $f \in X$ and $\|f\|_{X} \leq\|g\|_{X}$. Note that we omit the measure $\mu$ in the notation of the space $X(\mu)$ if the measure is clear in the context. We will say that $X$ is order continuous if for every $f, f_{n} \in X$ such that $0 \leq f_{n} \uparrow f \mu$-a.e., we have that $f_{n} \rightarrow f$ in the norm. The Köthe dual $X(\mu)^{\prime}$ of a Banach function space $X(\mu)$ is the subspace of $X(\mu)^{*}$ whose elements can be represented as integrals, that is $\varphi \in X(\mu)^{*}$ such that there is a function $g \in L^{0}(\mu)$ satisfying that $\varphi(f)=\int g f d \mu$ for every $f \in X(\mu)$. It is always an isometric subspace, and if $X(\mu)$ is $\sigma$-order continuous, then $X(\mu)^{*}=X(\mu)^{\prime}$.

Given two Banach function spaces $X$ and $Y$, the space of multiplication operators from $X$ to $Y$ is defined as the space of functions

$$
X^{Y}=\left\{h \in L^{0}(\mu): h f \in Y \text { for all } f \in X\right\},
$$

which always define multiplication operators $M_{h}: X \rightarrow Y$. Its norm is given by $\|\cdot\|_{X^{Y}}$ given by $\|h\|_{X^{Y}}=\sup _{f \in B_{X}}\|h f\|_{Y}$ for all $h \in X^{Y}$; in fact, it defines a natural seminorm on $X^{Y}$ which becomes a norm only in the case when $X^{Y}$ is saturated, that is, if there is a strictly positive function in the space. The reader can find some information about these spaces in $[1,11,17]$. They are a fundamental tool for factorization both of operators (see $[2,19,21])$ and of Banach function spaces (see $[12,23])$.

In this paper, the so called lattice tensor product or Fremlin tensor product of Banach lattices will be relevant. It is well-known that the projective tensor product of Banach lattices is not in general a Banach lattice. However, there is a construction that allows to assure that the corresponding completion of the tensor product has a 
Banach lattice structure. We are interested in the case when the Banach lattices involved are Banach function spaces. Consider two Banach function spaces $X(\mu)$ and $Y(\nu)$, and define the norm $|\pi|$ on the tensor product $X(\mu) \otimes Y(\nu)$ by

$$
|\pi|(z):=\inf \left\{\sum_{i=1}^{n}\left\|x_{i}\right\|\left\|y_{i}\right\|:|z| \leq \sum_{i=1}^{n}\left|x_{i}\right|\left|y_{i}\right|\right\} .
$$

Here, the order on the tensor product in the one inherited by the identification $(x, y) \mapsto$ $x(v) \cdot y(w) \in L^{0}(\mu \times \nu), x \in X, y \in Y$. The original construction can be found in [7], but the reader can find all what is needed for the present paper in [22].

Under certain $p$-convexity requirements on the spaces $X$ and $Y$, the norm $|\pi|$ can be computed in an easier way. Let us define

$$
\rho(u):=\inf \left\{\left\|x_{1}\right\| \cdot\left\|x_{2}\right\|:|u| \leq\left|x_{1}\right|\left|x_{2}\right|\right\}, \quad u \in X_{1} \otimes X_{2},
$$

and suppose that it is subadditive: in this case, it coincides with $|\pi|$ (see Theorem 2.1 in $[22])$. We write $X_{1} \hat{\otimes}_{|\pi|} X_{2}$ for the completion of $X_{1} \otimes_{|\pi|} X_{2}$.

We summarize in the following result some (almost) known properties of the lattice tensor product that will be needed in the paper.

Proposition 2.1. (Theorem 2.1 and Theorem 2.2 in [22]).

(i) If $\rho$ defined above on $X_{1} \otimes X_{2}$ is subadditive, then for each $u \in X_{1} \hat{\otimes}_{|\pi|} X_{2}$, there are $x_{1} \in X_{1}$ and $x_{2} \in X_{2}$ such that $|u| \leq\left|x_{1}\right|\left|x_{2}\right|$. Moreover, if $|\pi|(u) \leq 1$ and $\varepsilon>0$, $x_{1}$ and $x_{2}$ can be chosen such that $\left\|x_{1}\right\|\left\|x_{2}\right\|<1+\varepsilon$.

(ii) If $E$ is a Banach space, there is a one-to-one correspondence between positive bilinear maps $\varphi: X_{1} \times X_{2} \rightarrow E$ and their positive linearizations $T: X_{1} \hat{\otimes}_{|\pi|} X_{2} \rightarrow E$.

(iii) If $1<p<\infty$ and $X_{1}$ is $p$-convex and $X_{2}$ is $p^{\prime}$-convex - with convexity constants $M_{(p)}\left(X_{1}\right)=1$ and $M_{\left(p^{\prime}\right)}\left(X_{2}\right)=1$ - then $\rho$ is subadditive.

Proof. The main parts of the proofs of the different statements can be found in [22]. In (i), the norm estimate is not explicitly written in the proof of [22, Th.2.2(ii)]. However, a look to the argument shows that for a given $\varepsilon>0$, it is possible to find a convenient sequence $\left(u_{n}\right) \in X_{1} \otimes_{|\pi|} X_{2}$ approximating $u$ satisfying that $|\pi|\left(u_{n}\right) \leq 1$ and with $|\pi|\left(u_{n}-u_{n+1}\right)$ as small as we want. A standard bounding procedure gives the estimate.

(ii) is a direct consequence of $[22$, Th.2.2(iv)]. Finally, a look to the proof of $[22$, Th.2] - that is written for $L^{p}$-spaces - shows that it works for spaces with the adequate convexity requirements, giving (iii).

\section{Kernel representations of dominated operators}

In this section we introduce and study what we call integral domination inequalities for a pair of operators. This kind of inequalities consists of order relations among integrals involving the evaluations of both operators of the pair. For example, $p$-concavity of an operator $T: L^{p}(\mu) \rightarrow L^{p}(\mu)$ is given by

$$
\left(\sum_{i=1}^{n} \int\left|T\left(x_{i}\right)\right|^{p} d \mu\right)^{1 / p} \leq K\left(\int \sum_{i=1}^{n}\left|x_{i}\right|^{p} d \mu\right)^{1 / p},
$$


for all $x_{1}, \ldots, x_{n} \in L^{p}(\mu)$, that can be considered as an integral domination of $T$ by the identity map in $L^{p}$. Other classical example is given by some inequalities coming from classical harmonic analysis. To give a concrete example, the classical weighted Hardy inequalities

$$
\int_{0}^{\infty}\left(\frac{1}{s} \int_{0}^{s} x(t) d t\right)^{p} w(s) d s \leq c \int_{0}^{\infty} x(s)^{p} v(s) d s
$$

that hold for suitable Muckenhoupt weights $w(s)$ and $v(s)$ may be considered as an integral domination of the averaging operator by the identity map, and the reverse inequalities are in fact integral dominations of the identity by the averaging operator (see for example [18] and the references therein). We will come back to averaging type operators at the end of this section.

Let us start this part by introducing some technical definitions and results that will be necessary to provide the right theoretical context. Let $1<p<\infty$ and suppose that the spaces $X_{1}^{X_{2}}$ and $Y_{2}^{Y_{1}}$ are $p$-convex and $p^{\prime}$-convex, respectively. Consider the Fremlin tensor product of these spaces, that is,

$$
X_{1}^{X_{2}} \otimes_{|\pi|} Y_{2}^{Y_{1}} \text {. }
$$

We will say that a (closed) subspace $A$ of $\left(X_{1}^{X_{2}} \otimes_{|\pi|} Y_{2}^{Y_{1}}\right)^{*}$ is adequate if the weak topology $\tau_{A}$ defined by the duality $\left\langle X_{1}^{X_{2}} \otimes_{|\pi|} Y_{2}^{Y_{1}}, A\right\rangle$ separates points and satisfies that the unit ball of this Fremlin tensor product is compact for $\tau_{A}$. The canonical case that will be used in this paper is when $X_{1}^{X_{2}} \otimes_{|\pi|} Y_{2}^{Y_{1}}$ is a dual space and $A$ is its predual.

Consider two operators $T: X_{1}(\mu) \rightarrow Y_{1}(\nu)$ and $S: X_{2}(\mu) \rightarrow Y_{2}(\nu)$. Our first technical result provides a characterization of when the first operator can be factored through a kernel operator constructed from the second one in a particular way. The main requirement is given by the integral domination inequalities that appear in (ii) of the next lemma, which involve two operators.

Note first that, if $x_{1}, \ldots, x_{n} \in X_{1}$ and $y_{1}^{\prime}, \ldots, y_{n}^{\prime} \in Y_{1}^{\prime}$ and $S$ is as above, the expression

$$
\begin{gathered}
X_{1}^{X_{2}} \otimes Y_{2}^{Y_{1}} \ni \sum_{j=1}^{m} f_{j} \otimes g_{j} \rightsquigarrow\left(\sum_{i=1}^{n} S\left(\cdot x_{i}\right) \cdot\left(\cdot y_{i}^{\prime}\right)\right)\left(\sum_{j=1}^{m} f_{i} \otimes g_{i}\right) \\
:=\left\langle\sum_{i=1}^{n} S\left(\cdot x_{i}\right) \cdot\left(\cdot y_{i}^{\prime}\right), \sum_{j=1}^{m} f_{i} \otimes g_{i}\right\rangle=\sum_{i=1}^{n} \sum_{j=1}^{m} \int S\left(f_{j} x_{i}\right) \cdot\left(g_{j} y_{i}^{\prime}\right) d \mu \in \mathbb{R},
\end{gathered}
$$

defines a linear functional $\sum_{i=1}^{n} S\left(\cdot x_{i}\right) \cdot\left(\cdot y_{i}^{\prime}\right)$ in $\left(X_{1}^{X_{2}} \otimes Y_{2}^{Y_{1}}\right)^{*}$.

Lemma 3.1. Consider Banach function spaces $X_{1}(\mu)$ and $X_{2}(\mu)$ and $Y_{1}(\nu)$ and $Y_{2}(\nu)$, where $(\Omega, \Sigma, \mu)$ and $(\Delta, \Gamma, \nu)$ are $\sigma$-finite measure spaces. Suppose that both $X_{1}^{X_{2}}, Y_{2}^{Y_{1}}$ are saturated Banach function spaces. Suppose also that

$$
\left\{S(\cdot x) \cdot\left(\cdot y^{\prime}\right): y^{\prime} \in Y_{1}^{\prime}, x \in X_{1}\right\}
$$

lies in an adequate subspace $A$ of $\left(X_{1}^{X_{2}} \otimes_{|\pi|} Y_{2}^{Y_{1}}\right)^{*}$.

The following facts are equivalent for the operators $T: X_{1} \rightarrow Y_{1}$ and $S: X_{2} \rightarrow Y_{2}$.

(i) The inequality

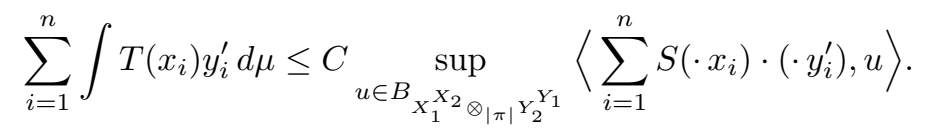


holds for every $x_{1}, \ldots, x_{n} \in X_{1}$ and $y_{1}^{\prime}, \ldots, y_{n}^{\prime} \in Y_{1}^{\prime}$.

(ii) There is an element $u_{0}$ in the unit ball of $X_{1}^{X_{2}} \otimes_{|\pi|} Y_{2}^{Y_{1}}$ such that

$$
\int T(x) y^{\prime} d \mu=C\left\langle S(\cdot x) \cdot\left(\cdot y^{\prime}\right), u_{0}\right\rangle,
$$

for all $x \in X_{1}$ and $y^{\prime} \in Y_{1}^{\prime}$.

Proof. We use the duality of the subspace $A$ with the lattice tensor product $X_{1}^{X_{2}} \otimes_{|\pi|} Y_{2}^{Y_{1}}$

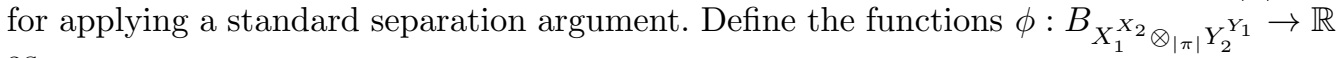
as

$$
\phi(u):=\sum_{i=1}^{n} \int T\left(x_{i}\right) y_{i}^{\prime} d \mu-C \sum_{i=1}^{n}\left\langle S\left(\cdot x_{i}\right) \cdot\left(\cdot y_{i}^{\prime}\right), u\right\rangle,
$$

where $x_{1}, \ldots, x_{n}$ and $y_{1}^{\prime}, \ldots, y_{n}^{\prime}$ are elements of $X_{1}$ and $Y_{1}^{*}$, respectively. It is easy to check that they form a concave class of convex functions that are continuous by hypothesis with respect to the weak topology defined by $A$ for $X_{1}^{X_{2}} \otimes_{|\pi|} Y_{2}^{Y_{1}}$. The inequalities in (i) and the Hahn-Banach Theorem gives a norm one element $u$ in the completion of the tensor product for each function $\phi$ such that $\phi(u) \leq 1$. Then, we have by Ky Fan's Lemma that there is an element $u_{0}$ in the completion of $X_{1}^{X_{2}} \otimes_{|\pi|} Y_{2}^{Y_{1}}$ of norm one such that

$$
\sum_{i=1}^{n} \int T\left(x_{i}\right) y_{i}^{\prime} d \mu \leq C \sum_{i=1}^{n}\left\langle S\left(\cdot x_{i}\right) \cdot\left(\cdot y_{i}^{\prime}\right), u_{0}\right\rangle .
$$

The element $u_{0}$ of the completion of the Fremlin tensor product satisfies that

$$
\int T(x) y^{\prime} d \mu \leq C\left\langle S(\cdot x) \cdot\left(\cdot y^{\prime}\right), u_{0}\right\rangle
$$

for all $x$ and $y^{\prime}$ and so, by changing $y^{\prime}$ by $-y^{\prime}$ we obtain

$$
-\int T(x) y^{\prime} d \mu \leq-C\left\langle S(\cdot x) \cdot\left(\cdot y^{\prime}\right), u_{0}\right\rangle
$$

that gives

$$
\int T(x) y^{\prime} d \mu=C\left\langle S(\cdot x) \cdot\left(\cdot y^{\prime}\right), u_{0}\right\rangle,
$$

for all $x$ and $y^{\prime}$. The converse is given by a direct computation.

Lemma 3.2. Let $X(\mu)$ and $Y(\mu)$ be Banach function spaces, and assume that $Y(\mu)$ is $\sigma$-order continuous. If $S: X(\mu) \rightarrow Y(\nu)$ is a positive operator and $u \in X \hat{\otimes}_{|\pi|} Y^{\prime}$, then there are $h \in X, g \in Y^{\prime}$ and $f \in B_{L^{\infty}(\mu \times \nu)}$ such that $u=h f g$ and

$$
\langle u, S\rangle=\int S(f(v, w) h(v)) g(w) d \nu .
$$

Moreover, if $u \in X \otimes_{|\pi|} Y^{\prime}$, then the operator is not needed to be positive for obtaining the same formula.

Proof. Note first that since $Y$ is $\sigma$-order continuous, we have that $Y^{*}$ can be identified with the Köthe dual $Y^{\prime}$. Take an element $u \in X \hat{\otimes}_{|\pi|} Y^{\prime}$ and consider a sequence of tensors $\left(u_{n}\right) \in X \otimes_{|\pi|} Y^{\prime}$ converging to $u$ with respect to $|\pi|$ and such that $|\pi|\left(u-u_{n}\right)<2^{-n}$. 
Take a representation of $u_{n}$ as $u_{n}=\sum_{i=1}^{m_{n}} x_{i} \otimes y_{i}^{\prime}$. It can be identified with the function $h_{n} f_{n} g_{n}$ of $L^{0}(\mu \times \nu)$ defined by $h_{n}(v)=\sum_{i=1}^{m_{n}}\left|x_{i}(v)\right|, g_{n}(w)=\sum_{i=1}^{m_{n}}\left|y_{i}^{\prime}(w)\right|$ and

$$
f_{n}(v, w)=\sum_{i=1}^{m_{n}} x_{i}(v) y_{i}^{\prime}(w) / h_{n}(v) g_{n}(w) .
$$

Note that, fixing $w$, we have that $h_{n}(v) f_{n}(v, w) g_{n}(w) \in X(\mu)$ and

$$
S\left(h_{n}(v) f_{n}(v, w) g_{n}(w)\right)=S\left(h_{n}(v) f_{n}(v, w)\right) \cdot g_{n}(w)=\sum_{i=1}^{m} S\left(x_{i}(v)\right) \cdot y_{i}^{\prime}(w) .
$$

Then

$$
\begin{gathered}
\left\langle u_{n}, S\right\rangle=\sum_{i=1}^{m}\left\langle S\left(x_{i}\right), y_{i}^{\prime}\right\rangle \\
=\sum_{i=1}^{m} \int S\left(x_{i}(v)\right)(w) y_{i}^{\prime}(w) d \nu(w)=\int S\left(h_{n}(v) f_{n}(v, w)\right) g_{n}(w) d \nu(w) .
\end{gathered}
$$

This gives the formula for the case of $u \in X \otimes_{|\pi|} Y^{\prime}$; no positivity of $S$ has been needed.

Let us see now the case when $u$ is an element of the completion. Recall that $u$ can be identified with an element of $L^{0}(\mu \times \nu)$, and that there are elements $x_{0} \in X$ and $y_{0}^{\prime} \in Y^{\prime}$ such that $|u(v, w)| \leq\left|x_{0}(v)\right|\left|y_{0}^{\prime}(w)\right| \mu \times \nu$-a.e. (Proposition 2.1(i)). For each fixed $w_{0}$, we get that $u\left(v, w_{0}\right)$ is $\mu$-measurable and $\left|u\left(v, w_{0}\right)\right| \leq\left|x_{0}(v)\right|\left|y_{0}^{\prime}\left(w_{0}\right)\right|$, and so $u\left(v, w_{0}\right) \in$ $X(\mu)$. As a consequence, the function $w_{0} \mapsto S\left(u\left(v, w_{0}\right)\right) \in Y^{*}=Y^{\prime}$ is well-defined. We can apply now Lebesgue's Dominated Convergence Theorem. In order to do that, let us consider the functions $w \mapsto S\left(u_{n}\right)(w):=S\left(u_{n}(v, w)\right)$ and $w \mapsto S(u)(w):=S(u(v, w))$. Recalling that the tensors $u_{u}$ have a simple representation, it is easy to compute explicitly $S\left(u_{n}\right)$ in order to see that the functions $S\left(u_{n}\right)$ are $\nu$-measurable.

1) By Proposition 2.1(i), for each $n$ there are elements $x_{n} \in X$ and $y_{n}^{\prime} \in Y^{*}$ such that $\left|S\left(u_{n}\right)(w)-S(u)(w)\right| \leq\left|x_{n}(v)\left\|y_{n}^{\prime}(w) \mid,\right\| x_{n} \|<2^{-n / 2}\right.$ and $\left\|y_{n}^{\prime}\right\|<2^{-n / 2}$. We can assume without loss of generality that $x_{n} \rightarrow 0$ and $y_{n}^{\prime} \rightarrow 0$ a.e., since every converging sequence in a Banach function space has a subsequence that converges a.e. Then, since $S$ is positive, we have that for a fixed $w_{0}$,

$$
\begin{gathered}
\left|S\left(u_{n}\right)\left(w_{0}\right)-S(u)\left(w_{0}\right)\right|=\left|S\left(u_{n}\left(v, w_{0}\right)\right)-S\left(u\left(v, w_{0}\right)\right)\right| \\
\leq S\left(\left|u_{n}\left(v, w_{0}\right)-u\left(v, w_{0}\right)\right|\right) \leq S\left(\left|x_{n}(v)\right|\left|y_{n}^{\prime}\left(w_{0}\right)\right|\right) \\
\leq S\left(\sum_{n \geq 1}\left|x_{n}(v)\right|\left|y_{n}^{\prime}\left(w_{0}\right)\right|\right)=S\left(\sum_{n \geq 1}\left|x_{n}(v)\right|\right)\left(w_{0}\right) \cdot\left|y_{n}^{\prime}\left(w_{0}\right)\right| .
\end{gathered}
$$

Observe that $\sum_{n \geq 1}\left|x_{n}\right| \in X$ as a consequence of the fact that $\left(x_{n}\right)_{n}$ converges absolutely, and so $S\left(\sum_{n \geq 1}\left|x_{n}\right|\right)$ is a well defined function of $w$ belonging to $Y$. Since $y_{n}^{\prime}$ tends to 0 a.e., we obtain that $S\left(u_{n}\right) \rightarrow S(u)$ a.e. 
2) For a fixed $n$, we have that

$$
\begin{gathered}
\left|S\left(u_{n}\right)(w)\right| \leq\left|S\left(u_{n}\right)(w)-S(u)(w)\right|+|S(u)(w)| \\
\leq S\left(\sum_{n \geq 1}\left|x_{n}\right|\right)(w) \cdot\left(\sum_{n \geq 1}\left|y_{n}^{\prime}\right|(w)\right)+S\left(x_{0}\right)(w) \cdot y_{0}^{\prime}(w) \in L^{1}(\nu) .
\end{gathered}
$$

Since the function dominating does not depend on $n$, we can apply the Dominated Convergence Theorem to obtain that $S(u)(w) \in L^{1}(\nu)$ and

$$
\begin{gathered}
\langle u, S\rangle=\lim _{n}\left\langle u_{n}, S\right\rangle=\lim _{n} \int S\left(u_{n}\right)(w) d \nu(w) \\
=\int S(u)(w) d \mu_{2}(w)=\int S\left(x_{0}(v) f_{0}(v, w)\right) y_{0}^{\prime}(w) d \nu(w),
\end{gathered}
$$

where $u=x_{0}(v) f_{0}(v, w) y_{0}^{\prime}(w)$ with

$$
f_{0}(v, w):=\frac{u(v, w)}{x_{0}(v) y_{0}^{\prime}(w)} \in B_{L^{\infty}\left(\mu_{1} \times \mu_{2}\right)} .
$$

This gives the result.

Theorem 3.3. Suppose that $X_{1}^{X_{2}}$ and $Y_{2}^{Y_{1}}$ are saturated Banach function spaces over $\mu_{1}$ and $\mu_{2}$, respectively, and that $\rho$ is subadditive on $X_{1}^{X_{2}} \otimes Y_{2}^{Y_{1}}$. Assume that $Y_{2}^{Y_{1}}$ is the dual of a $\sigma$-order continuous Banach function space and $Y_{1}$ is $\sigma$-continuous. Suppose also that

$$
\left\{S(\cdot x) \cdot\left(\cdot y^{\prime}\right): y^{\prime} \in Y_{1}^{\prime}, x \in X_{1}\right\}
$$

lies in an adequate subspace $A$ of $\left(X_{1}^{X_{2}} \otimes_{|\pi|} Y_{2}^{Y_{1}}\right)^{*}$. The following facts are equivalent for the positive operator $T: X_{1} \rightarrow Y_{1}$ and the operator $S: X_{2} \rightarrow Y_{2}$.

(i) The inequality

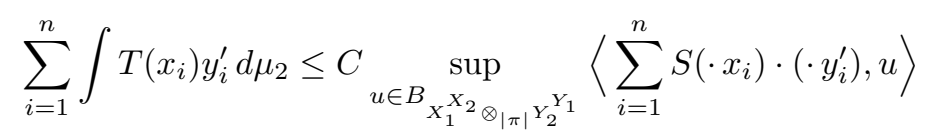

holds for every $x_{1}, \ldots, x_{n} \in X_{1}$ and $y_{1}^{\prime}, \ldots, y_{n}^{\prime} \in Y_{1}^{\prime}$.

(ii) There are a function $h\left(w_{1}\right) \in B_{X_{1}^{X_{2}}}$, a function $g\left(w_{2}\right) \in B_{Y_{2}^{Y_{1}}}$ and a bounded function $f\left(w_{1}, w_{2}\right) \in L^{\infty}\left(\mu_{1} \times \mu_{2}\right)$ such that $g f h \in X_{1}^{X_{2}} \hat{\otimes}_{|\pi|} Y_{2}^{Y_{1}{ }^{2}}$ and

$$
T(x)=g \hat{S}(h x)
$$

for all $x \in X_{1}$, where $\hat{S}: X_{2}\left(\mu_{1}\right) \rightarrow Y_{2}\left(\mu_{2}\right)$ is a kernel-type operator associated to the function $f(v, w)$ by

$$
\hat{S}(z)(w)=S(z(v) f(v, w)), \quad z \in X_{2}\left(\mu_{1}\right) .
$$


That is, if (i) or (ii) hold, then $T$ factors through the kernel operator $\hat{S}$ defined above as

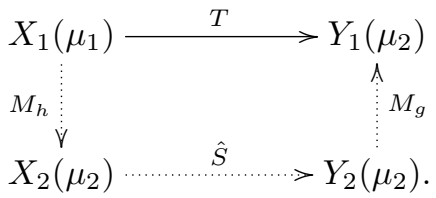

Moreover, if $X_{1}^{X_{2}} \otimes_{|\pi|} Y_{2}^{Y_{1}}$ is already complete, the positivity of $S$ is not needed.

Proof. Let as see that (i) implies (ii). The proof is a consequence of Theorem 3.1. By assumption, the norm for an element $u$ of the Fremlin tensor product $X_{1} \otimes_{|\pi|} X_{2}$ (considered as a subspace of $\left.L^{0}\left(\mu_{1} \times \mu_{2}\right)\right)$ can be computed by

$$
\|u\|_{|\pi|}=\inf \left\{\left\|x_{1}\right\|\left\|x_{2}\right\|:|u| \leq\left|x_{1}\right|\left|x_{2}\right|\right\},
$$

and by Proposition 2.1(i) each element of $X_{1}^{X_{2}} \hat{\otimes}_{|\pi|} Y_{2}^{Y_{1}}$ can be dominated by a single product of elements of $X_{1}^{X_{2}}$ and $Y_{2}^{Y_{1}}$ with convenient norms. This implies that for the norm one element $u_{0}$ provided in the equality (ii) of Theorem 3.1 and $\varepsilon>0$, there are functions $h$ and $g$ such that $\|h\|<1+\varepsilon$ and $\|g\|=1$ such that $u_{0} \in X_{1}^{X_{2}} \otimes_{|\pi|} Y_{2}^{Y_{1}}$ and

$$
\left|u_{0}(v, w)\right| \leq|h(v)||g(v)|
$$

as elements of $L^{0}\left(\mu_{1} \times \mu_{2}\right)$. This gives that $u_{0}$ can be written as

$$
u_{0}(v, w)=\frac{u_{0}(v, w)}{|h(v)||g(w)|}|h(v)||g(w)|,
$$

and so it is written as a product of the function $f_{0}:=\frac{u_{0}}{|h||g|}$ in $B_{L^{\infty}}\left(\mu_{1} \times \mu_{2}\right)$ and two functions $h$ and $g$ belonging to $(1+\varepsilon) B_{X_{1}^{X_{2}}}$ and $B_{Y_{2}^{Y_{1}}}$, respectively. Recall also that $Y_{1}$ is assumed to be $\sigma$-order continuous, and so $Y_{1}^{*}=Y_{1}^{\prime}$.

Thus, we have that for $x \in X_{1}$ and $y^{\prime} \in Y_{1}^{*}=Y_{1}^{\prime}$,

$$
\begin{gathered}
\int T(x) y^{\prime} d \mu_{2}=\left\langle S(\cdot x) \cdot\left(\cdot y^{\prime}\right), u_{0}\right\rangle=\left\langle S(\cdot x) \cdot\left(\cdot y^{\prime}\right), h(v) f(v, w) g(w)\right\rangle \\
=\left\langle S, h(v) x(v) f(v, w) g(w) y^{\prime}(w)\right\rangle .
\end{gathered}
$$

By Lemma 3.2, and taking into account that $Y_{2}^{Y_{1}}$ is assumed to be the dual of a $\sigma$-order continuous lattice, we obtain

$$
\int T(x) y^{\prime} d \mu_{2}=\int S(h(v) x(v) f(v, w)) g(w) y^{\prime}(w) d \mu_{2} .
$$

This clearly gives the desired factorization.

For (ii) $\Rightarrow$ (i), just note that by assumption the elements $h \in X_{1}^{X_{2}}, f \in L^{\infty}\left(\mu_{1}, \mu_{2}\right)$ and $g \in Y_{2}^{Y_{1}}$ defines a function $g f h$ that is an element of the closure of $X_{1}^{X_{2}} \otimes_{|\pi|} Y_{2}^{Y_{1}}$. 


\subsection{An example: $A, k_{A}$-averaging operators}

Let us finish this section by showing a concrete application of Theorem 3.3 to the case of the averaging operators (conditional expectation operators) on Banach function spaces associated to a given partition of a measure space. This class of operators constitutes a basic tool in functional analysis, and has been used both for applications and for theoretical purposes. Starting with the classic paper [20] by G.C. Rota (see also the references in it), the reader can find some information on this topic in the setting of the Riesz spaces and the Banach function spaces in $[13,5]$.

Take a finite measure space $(\Omega, \Sigma, \mu)$ and a finite partition $A:=\left\{A_{k}: k=1, \ldots, s\right\}$ of $\Omega$ defined by sets of positive measure. Let $1<p<\infty$. Consider a kernel function $k_{A}$ : $\Omega \times \Omega \rightarrow \mathbb{R}$ defining an operator $K_{A}: L^{p}(\mu) \rightarrow L^{p}(\mu)$ by $K_{A}(x)=\int k_{A}(v, w) z(v) d \mu(v)$, $z \in L^{p}(\mu)$.

Let us define the $A, k_{A}$-averaging kernel operator $E_{A, k_{A}}: L^{p}(\mu) \rightarrow L^{p}(\mu)$ given by

$$
E_{A, k_{A}}(z)(w):=\sum_{k=1}^{n}\left(\int_{A_{k}} k_{A}(v, w) z(v) d \mu(v)\right) \frac{\chi_{A_{k}}(w)}{\mu\left(A_{k}\right)},
$$

$z \in L^{p}(\mu)$, and assume that it is well-defined and continuous. For example, for the kernel given by the constant 1 function, it gives the classical example of a is well-defined and continuous operator (see for example [20, S.2]). We will write simply $E_{A}$ for this operator, that is $E_{A}:=E_{A, 1}$. Take Banach function spaces $X(\mu)$ and $Y(\mu)$ such that $X(\mu) \subseteq L^{p}(\mu)$ and $L^{p}(\mu) \subseteq Y(\mu)$. Note that by Lemma 5.1 in [1], $X^{L^{p}}$ is $p$-convex and $\left(L^{p}\right)^{Y}=\left(Y^{\prime}\right)^{L^{p^{\prime}}}$ is $p^{\prime}$-convex with constants equal to 1. By Proposition 2.1(iii), we have that $\rho$ is subadditive.

In this case we have that for $x_{i} \in X, h_{j} \in X^{L^{p}}, y_{i}^{\prime} \in Y^{\prime}$ and $g_{j} \in\left(L^{p}\right)^{Y}$,

$$
\begin{gathered}
\left\langle\sum_{i=1}^{n} E_{A}\left(\cdot x_{i}\right) \cdot\left(\cdot y_{i}^{\prime}\right), \sum_{j=1}^{m} h_{j} \otimes g_{j}\right\rangle \\
=\sum_{i=1}^{n} \sum_{j=1}^{m} \int E_{A}\left(h_{j} x_{i}\right) \cdot\left(g_{j} y_{i}^{\prime}\right) d \mu=\sum_{i=1}^{n} \sum_{j=1}^{m} \sum_{k=1}^{s}\left(\int_{A_{k}} h_{j} x_{i}, d \mu\right)\left(\int \frac{\chi_{A_{k}}}{\mu\left(A_{k}\right)} g_{j} y_{i}^{\prime} d \mu\right) \\
=\sum_{i=1}^{n} \sum_{j=1}^{m} \sum_{k=1}^{s} \frac{\left(\int_{A_{k}} h_{j} x_{i}, d \mu\right)\left(\int_{A_{k}} g_{j} y_{i}^{\prime} d \mu\right)}{\mu\left(A_{k}\right)} .
\end{gathered}
$$

Therefore, the requirement appearing in Theorem 3.3(i) for an operator $T: X(\mu) \rightarrow$ $Y(\mu)$ is that the inequality

$$
\begin{gathered}
\sum_{i=1}^{n} \int T\left(x_{i}\right) y_{i}^{\prime} d \mu \\
\leq C \sup _{\sum_{j=1}^{m} h_{j} \otimes g_{j} \in B_{X L^{p} \otimes_{|\pi|}\left(L^{p}\right)^{Y}}}\left\{\sum_{i=1}^{n} \sum_{j=1}^{m} \int E_{A}\left(h_{j} x_{i}\right) \cdot\left(g_{j} y_{i}^{\prime}\right) d \mu\right\}
\end{gathered}
$$


holds for every $x_{1}, \ldots, x_{n} \in X$ and $y_{1}^{\prime}, \ldots, y_{n}^{\prime} \in Y^{\prime}$. This is equivalent to the inequality

$$
\begin{gathered}
\sum_{i=1}^{n} \int T\left(x_{i}\right) y_{i}^{\prime} d \mu \\
\leq C \sup _{\sum_{j=1}^{m} h_{j} \otimes g_{j} \in B_{X^{L^{p}} \otimes_{|\pi|}\left(L^{p}\right)^{Y}}}\left\{\sum_{k=1}^{s} \sum_{i=1}^{n} \sum_{j=1}^{m} \frac{\left(\int_{A_{k}} h_{j} x_{i}, d \mu\right)\left(\int_{A_{k}} g_{j} y_{i}^{\prime} d \mu\right)}{\mu\left(A_{k}\right)}\right\} .
\end{gathered}
$$

Assume that $\left(L^{p}\right)^{Y}$ is the dual of a $\sigma$-order continuous Banach function space and $Y$ is $\sigma$-order continuous. Thus, if the inequality above holds for $T$, Theorem 3.3 (for $\nu=\mu$ ) gives that there are functions $h_{A} \in B_{X^{L^{p}}}, g_{A} \in B_{\left(L^{p}\right)^{Y}}$ and $f_{A} \in L^{\infty}(\mu \times \mu)$ such that

$$
T(x)=g_{A} \hat{E}_{A}(h x)
$$

for all $x \in X$, where $\hat{E_{A}}: L^{p}(\mu) \rightarrow L^{p}(\mu)$ is exactly the averaging kernel operator given by

$$
\begin{gathered}
\hat{E_{A}}(z)(w)=E_{A}\left(z(v) f_{A}(v, w)\right) \\
=\sum_{k=1}^{n}\left(\int_{A_{k}} z(v) f_{A}(v, w) d \mu(v)\right) \frac{\chi_{A_{k}}(w)}{\mu\left(A_{k}\right)}=E_{A, f_{A}}(z), \quad z \in L^{p}(\mu) .
\end{gathered}
$$

That is,

$$
T(x)=g(w)\left[\sum_{k=1}^{n} \frac{\chi_{A_{k}}(w)}{\mu\left(A_{k}\right)}\left(\int_{A_{k}} x(v) h(v) f_{A}(v, w) d \mu(v)\right)\right]
$$

for all $x \in X(\mu)$. The converse is obvious: if $g, f$ and $h$ are as above and $g f h \in$ $X^{L^{p}} \hat{\otimes}_{|\pi|}\left(L^{p}\right)^{Y}$, then the operator

$$
T(x)(w):=g(w) E_{A}\left(f_{A}(v, w) h(v) x(v)\right), \quad x \in X(\mu),
$$

that allows an expression as the one above, satisfies the inequality (3.2). This gives the following generalized version of the approximation of the identity map by means of integral averages for order continuous Banach function spaces. Let $(\Omega, \Sigma, \mu)$ be a finite measure space and consider the ordered directed set $\mathcal{A}$ of all $\mu$-a.e. equal finite measurable partitions of $\Omega$, where the order is given by: $A_{1} \leq A_{2}$ if and only if $A_{2}$ is finer than $A_{1}$ $\mu$-a.e.

Corollary 3.4. Let $X(\mu)$ and $Y(\mu)$ be Banach function spaces such that $\left(L^{p}(\mu)\right)^{Y}$ is the dual of a $\sigma$-order continuous Banach function space and $Y$ is $\sigma$-order continuous. Suppose that the operator $T: X(\mu) \rightarrow Y(\mu)$ is the pointwise limit of a net $\left\{T_{A}: A \in \mathcal{A}\right\}$, each one satisfying a domination as

$$
\sum_{i=1}^{n} \int T_{A}\left(x_{i}\right) y_{i}^{\prime} d \mu \leq \sup _{h \in B_{X^{L}}, g \in B_{\left(L^{p}\right)^{Y}}}\left\{\sum_{k=1}^{s} \sum_{i=1}^{n} \frac{\left(\int_{A_{k}} h x_{i} d \mu\right)\left(\int_{A_{k}} g y_{i}^{\prime} d \mu\right)}{\mu\left(A_{k}\right)}\right\}
$$

for every $x_{1}, \ldots, x_{n} \in X_{1}$ and $y_{1}^{\prime}, \ldots, y_{n}^{\prime} \in Y_{1}^{\prime}$. Then for each $x \in X$,

$$
T(x)(w)=\lim _{A}\left(g_{A}(w) \cdot E_{A, f_{A}}\left(h_{A}(v) x(v)\right)(w)\right)
$$

where $h_{A} \in B_{X^{L^{p}}}, g_{A} \in B_{\left(L^{p}\right)^{Y}}$ and $f_{A} \in B_{L^{\infty}(\mu \times \mu)}$ for each finite partition $A$. 
The canonical examples of Banach function spaces satisfying al the requirements on $X(\mu)$ and $Y(\mu)$ are $X=L^{r}[0,1]$ and $Y=L^{s}[0,1]$ for $1 \leq s<p \leq r<\infty$, being $\mu$ Lebesgue measure on $[0,1]$, or Lorentz spaces $L^{r, t}[0,1]$ and $L^{s, q}[0,1]$ satisfying the inclusion requirements with respect to $L^{p}[0,1]$.

\section{Trigonometric domination of operators}

We will apply the results of the previous sections for the case of trigonometric dominations by considering the following vector norm inequality for an operator $T$ between Hilbert spaces. Recall that we write $\mathcal{T}=\left\{t_{k}: k \in \mathbb{N}\right\}$ in $L^{2}[0,1]$ for the normalized trigonometric system. We will say that an operator $T: L^{2}(\mu) \rightarrow \ell^{2}$ satisfies a trigonometric domination if for each finite subset $J \subseteq \mathbb{N}$ we have that

$$
\sum_{i=1}^{n} \sum_{j \in J} T\left(x_{i}\right)_{j} a_{i, j} \leq C_{J} \sup _{\left(h_{j}\right)_{j \in J} \subset B_{L^{\infty}}}\left(\sum_{i=1}^{n} \sum_{j \in J}\left(\int h_{j} x_{i} t_{j} d \mu\right) a_{i, j}\right)
$$

holds for every $x_{1}, \ldots, x_{n} \in L^{2}[0,1]$ and real matrices $\left(a_{i, j}\right)_{i=1, \ldots, n, j \in J}$.

Our technique would work also for example for the case when $2 \leq p<\infty$ and $T$ is an operator $T: L^{p}[0,1] \rightarrow \ell^{p}$, since $L^{p}[0,1] \subseteq L^{2}[0,1]$ and $\ell^{2} \subseteq \ell^{p}$. However, we center our attention in the simplest case $p=2$, for which an easy characterization of our class of operators is provided.

Let us represent first in a concise way the elements that appear in this type of domination. Since the operators have finite dimensional range, we can write the lattice tensor product that appears in this case as follows, taking into account that $\left(L^{2}[0,1]\right)^{L^{2}[0,1]}=L^{\infty}[0,1]$ and $\left(\ell_{m}^{2}\right)^{\ell_{m}^{2}}=\ell_{m}^{\infty}$. Indeed, we have

$$
X_{1}^{X_{2}} \otimes_{|\pi|} Y_{1}^{Y_{2}}=L^{\infty}[0,1] \otimes_{|\pi|} \ell_{m}^{\infty} .
$$

Note that both spaces defining the tensor product are 2-convex with constants equal to 1 , and so by Proposition 2.1(iii) the norm can be defined by the function $\rho$, since it is subadditive.

Lemma 4.1. The lattice $L^{\infty}[0,1] \otimes_{|\pi|} \ell_{m}^{\infty}$ is isomorphic topologically and in the order to the $m$-fold Cartesian product

$$
L^{\infty}[0,1] \times \underbrace{\cdots}_{m} \times L^{\infty}[0,1]
$$

with its natural order and the norm $\left\|\left(h_{1}, \ldots, h_{m}\right)\right\|=\max _{k=1, \ldots, m}\left\|h_{k}\right\|_{L^{\infty}[0,1]}$. Moreover,

$$
\left\|\left(h_{1}, \ldots, h_{m}\right)\right\| \leq|\pi|\left(\sum_{k=1}^{m} h_{k} \otimes e_{k}\right) \leq m \cdot\left\|\left(h_{1}, \ldots, h_{m}\right)\right\|
$$

for each $\left(h_{1}, \ldots, h_{m}\right) \in L^{\infty}[0,1] \times \cdots \times L^{\infty}[0,1]$.

In what follows sometimes we will write $L^{\infty}[0,1] \times L^{\infty}[0,1]$ for the $m$-fold Cartesian product and $L^{\infty}$ instead of $L^{\infty}[0,1]$ for the aim of clarity. 
Proof. Each element in $L^{\infty}[0,1] \times \cdots \times L^{\infty}[0,1]$ have a unique representation $(\mu$-a.e in the left part of the tensors) as $z=\sum_{k=1}^{m} h_{k} \otimes e_{k}$, where $e_{k}$ are the elements of the standard basis of $\ell_{m}^{\infty}$. We identify $z$ with the function $\left(h_{1}, \ldots, h_{m}\right) \in L^{\infty}[0,1] \times \cdots \times L^{\infty}[0,1]$.

Suppose that $|\pi|(z)=1$. Then for $\varepsilon>0$ we have a pair of elements $f_{z} \in L^{\infty}[0,1]$ and $\lambda_{z}=\left(\lambda_{k}\right)_{k=1}^{m} \in \ell_{m}^{\infty}$ such that

$$
\left|\sum_{k=1}^{m} h_{k} \cdot e_{k}\right| \leq\left|f_{z}\right|\left|\left(\lambda_{k}\right)\right|
$$

and $\left\|f_{z}\right\| \cdot\left\|\lambda_{z}\right\|<1+\varepsilon$. We can assume w.l.o.g that $\left\|\lambda_{z}\right\|=1$. Then

$$
\left\|\left(h_{1}, \ldots, h_{m}\right)\right\|_{L^{\infty}[0,1] \times \cdots \times L^{\infty}[0,1]}=\max _{k=1, \ldots, m}\left\|f_{k}\right\|_{L^{\infty}[0,1]} \leq\left\|f_{z}\right\| \cdot\left\|\lambda_{z}\right\|<1+\varepsilon .
$$

For the converse inequality, just note that

$$
\left|\sum_{k=1}^{m} h_{k} \cdot e_{k}\right| \leq\left(\sum_{k=1}^{m}\left|h_{k}\right|\right) \cdot\left|\sum_{k=1}^{m} e_{k}\right|
$$

that gives

$$
|\pi|\left(\sum_{k=1}^{m} h_{k} \cdot e_{k}\right) \leq\left\|\sum_{k=1}^{m}\left|h_{k}\right|\right\|_{L^{\infty}} \cdot\left\|\sum_{k=1}^{m} e_{k}\right\|_{\ell_{m}^{\infty}} \leq \sum_{k=1}^{m}\left\|h_{k}\right\|_{L^{\infty}} \leq m \cdot \max _{k=1, \ldots, m}\left\|f_{k}\right\|_{L^{\infty}} .
$$

Consider now the Fourier operator $\mathcal{F}: L^{2}[0,1] \rightarrow \ell^{2}$. Write $c$ for the counting measure on $\mathbb{N}$. We will develop an approximation procedure, so we consider the finite rank operators $T_{J}:=P_{J} \circ T$ defined by the finite dimensional projection $P_{J}: \ell^{2} \rightarrow \ell^{2}$ given by a finite subset $J \subseteq \mathbb{N}$ of subindexes referred to the corresponding elements of the trigonometric basis $\mathcal{T}$ of $L^{2}[0,1]$. The dominating operator is given by

$$
\mathcal{F}_{J}(x):=\sum_{j \in J}\left\langle x, t_{j}\right\rangle e_{j}, \quad x \in L^{2}[0,1] .
$$

Then, and taking into account the particular descriptions of the elements involved, the domination inequality that must be considered in this case is given by the following expression, where $x_{1}, \ldots, x_{n} \in L^{2}[0,1]$ and $y^{1}, \ldots, y^{n} \in \ell^{\infty}$, with $y^{i}=\left(y_{j}^{i}\right)_{j=1}^{m}$.

$$
\begin{gathered}
\sum_{i=1}^{n} \int P_{J} \circ T\left(x_{i}\right) y^{i} d c \\
\leq C_{J} \sup _{\sum_{k \in J} h_{k} \otimes e_{k} \in B_{L} \infty \otimes_{|\pi|} \ell^{\infty}}\left(\sum_{i=1}^{n} \sum_{j \in J} \sum_{k \in J}\left\langle h_{k} x_{i}, t_{j}\right\rangle\left\langle e_{j}, e_{k} \cdot y^{i}\right\rangle\right) \\
\leq C_{J} \sup _{\left(h_{j}\right)_{j \in J} \subset B_{L} \infty}\left(\sum_{i=1}^{n} \sum_{j \in J}\left\langle h_{j} x_{i}, t_{j}\right\rangle y_{j}^{i}\right),
\end{gathered}
$$

for certain constants $C_{J}$. We can get directly the equivalent expression

$$
\sum_{i=1}^{n} \sum_{j \in J} T\left(x_{i}\right)_{j} y_{j}^{i} \leq C_{J} \sup _{\left(h_{j}\right)_{j \in J} \subset B_{L^{\infty}}}\left(\sum_{i=1}^{n} \sum_{j \in J} \alpha_{j}\left(h_{j} x_{i}\right) y_{j}^{i}\right),
$$

where $\alpha_{j}\left(h_{j} x_{i}\right)=\int h_{j} x_{i} t_{j} d \mu, j \in J$, is the $j$-th Fourier coefficient of the function $h_{j} x_{i}$. 
Theorem 4.2. Let $J \subseteq \mathbb{N}$ be a finite subset. The following statements are equivalent for an operator $T: L^{2}[0,1] \rightarrow \ell^{2}$.

(i) T satisfies a trigonometric domination, that is, for every finite subset $J \subset \mathbb{N}$, there is a constant $C_{J}$ such that the inequality

$$
\sum_{i=1}^{n} \sum_{j \in J} T\left(x_{i}\right)_{j} a_{i, j} \leq C_{J} \sup _{\left(h_{j}\right)_{j \in J} \subset B_{L} \infty}\left(\sum_{i=1}^{n} \sum_{j \in J}\left(\int h_{j} x_{i} t_{j} d \mu\right) a_{i, j}\right)
$$

holds for every $x_{1}, \ldots, x_{n} \in L^{2}[0,1]$ and matrices $\left(a_{i, j}\right)_{i=1, \ldots, n, j \in J}$.

(ii) There are functions $h_{j}^{J} \in L^{\infty}[0,1]$ with $\left\|h_{j}^{J}\right\| \leq C_{J}, j \in J$, such that

$$
P_{J} \circ T(x)=\left(\alpha_{j}\left(h_{j}^{J} x\right)\right)_{j \in J}=\left(\int h_{j}^{J} x t_{j} d \mu\right)_{j \in J} .
$$

(iii) For each finite subset $J \subset \mathbb{N}, P_{J} \circ T$ factors through a perturbation $\hat{\mathcal{F}}_{J}$ of the Fourier transform as

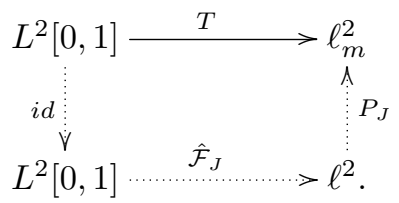

where $\hat{\mathcal{F}}_{J}(x)=\sum_{j \in J}\left(\int h_{j}^{J} x t_{j} d \mu\right) e_{j}$ and $h_{j}^{J} \in L^{\infty}[0,1]$ with $\left\|h_{j}^{J}\right\| \leq C_{J}, j \in J$.

Proof. Let as see (i) implies (ii). The proof is a consequence of the computations above and Theorem 3.3. Note first that $L^{\infty}[0,1]$ and $\ell^{\infty}$ are 2 -convex with constants 1 , and then by Lemma 2.1(iii), $\rho$ is subadditive. By Lemma 4.1 the Fremlin tensor product is (isomorphic to) the dual of the $m$-fold Cartesian product $L^{1}[0,1] \times \cdots \times L^{1}[0,1]$ with the $\ell^{1}$-sum, which provides the admisibility requirement that is needed for the dual pair. Then we are in position of applying Theorem 3.3, since $\ell^{2}$ is $\sigma$-order continuous and $\left(\ell^{2}\right)^{\ell^{2}}=\ell^{\infty}$, that is the dual of the $\sigma$-order continuous space $\ell^{1}$. It gives a factorization of $P_{J} \circ T$ as

$$
P_{J} \circ T(x)=g(w) \mathcal{F}(f(v, w) h(v) x(v)),
$$

where $g$ is a sequence in $\ell_{m}^{\infty}, h \in L^{\infty}[0,1]$ and $f \in L^{\infty}\left(\mu \times\left. c\right|_{J}\right)$, for all $x \in L^{2}$. In this case, we can identify $f(v, w) h(v)$ with the finite sequence $\left(h_{j}^{J}(v)\right)_{j \in J}$ of functions of $L^{\infty}[0,1]$, in such a way that $\max _{j \in J}\left\|h_{j}\right\| \leq C_{J}$, and $g$ with the constant sequence (1) $j \in J$. Therefore,

$$
P_{J} \circ T(x)=\left(\int h_{j}^{J} x t_{j} d \mu\right)_{j \in J}, \quad x \in L^{2}[0,1] .
$$

This gives (ii). The converse is given by a direct computation, and clearly (ii) and (iii) are equivalent. This finishes the proof.

Let us finish by showing our explicit characterization of weighted Fourier operators in $L^{2}[0,1]$. In fact, we implicitly show that the domination inequality can be simplified in Theorem 4.2(i) and rewritten in terms of the Fourier coefficients of the functions involved. 
We say that an operator $T: L^{2}[0,1] \rightarrow L^{2}[0,1]$ is finitely dominated by $\mathcal{F}$ if for each finite subset $J \subset \mathbb{N}$ there is a constant $C_{J}$ such that

$$
\sum_{i=1}^{n} \sum_{j \in J}\left(\int T\left(x_{i}\right) t_{j} d \mu\right) a_{i, j} \leq C_{J} \sup _{\left(h_{j}\right)_{j \in J} \subset B_{L^{\infty}}}\left(\sum_{i=1}^{n} \sum_{j \in J}\left(\int h_{j} x_{i} t_{j} d \mu\right) a_{i, j}\right)
$$

holds for every $x_{1}, \ldots, x_{n} \in L^{2}[0,1]$ and matrices $\left(a_{i, j}\right)_{i=1, \ldots, n, j \in J}$.

The same inequality can be written as a domination of Fourier coefficients associated to $T(x)$ by Fourier coefficients associated to $h x$, where $h$ is a function of $L^{\infty}[0,1]$. Indeed, this is equivalent to the fact that for each finite subset $J \subset \mathbb{N}$ there is a constant $C_{J}$ such that

$$
\sum_{i=1}^{n} \sum_{j \in J} \alpha_{j}\left(T\left(x_{i}\right)\right) a_{i, j} \leq C_{J} \sup _{\left(h_{j}\right)}\left(\sum_{j \in J} \subset B_{L} \sum_{i=1} \alpha_{j}\left(h_{j} x_{i}\right) a_{i, j}\right)
$$

holds for every $x_{1}, \ldots, x_{n} \in L^{2}[0,1]$ and matrices $\left(a_{i, j}\right)_{i=1, \ldots, n, j \in J}$.

Corollary 4.3. The following statements are equivalent for an operator $T: L^{2}[0,1] \rightarrow$ $L^{2}[0,1]$.

(i) $T$ is finitely dominated by $\mathcal{F}$.

(ii) For each $j \in \mathbb{N}$ there is a constant $C_{j}$ such that

$$
\sum_{i=1}^{n} \alpha_{j}\left(T\left(x_{i}\right)\right) \leq C_{j} \cdot \sup _{h \in B_{L^{\infty}}}\left(\sum_{i=1}^{n} \alpha_{j}\left(h x_{i}\right)\right)
$$

holds for every finite set $x_{1}, \ldots, x_{n} \in L^{2}[0,1], n \in \mathbb{N}$.

(iii) For each $j \in \mathbb{N}$ there is a constant $C_{j}$ such that

$$
\alpha_{j}(T(x)) \leq C_{j} \int\left|t_{j}\right||x| d \mu
$$

for all $x \in L^{2}[0,1]$.

(iv) There is a sequence $\left(h_{j}\right)_{j \geq 1} \subseteq L^{\infty}[0,1]$ such that

$$
T(x)(w)=\sum_{j=1}^{\infty}\left(\int h_{j} x t_{j} d \mu\right) t_{j}(w)
$$

for each $x \in L^{2}[0,1]$, that is, $T$ is a weighted Fourier operator.

Proof. Obviously, (i) implies (ii), and (ii) implies (iii). For showing that (iii) implies (ii), take a sequence $x_{1}, \ldots, x_{n} \in L^{2}[0,1]$. Then the linearity of all the involved expressions in (iii) gives (ii), since

$$
\begin{gathered}
\sum_{i=1}^{n} \alpha_{j}\left(T\left(x_{i}\right)\right) \leq C_{j} \int\left|t_{j}\right|\left|\sum_{i=1}^{n} x_{i}\right| d \mu \\
=C_{j} \sup _{h \in B_{L^{\infty}}}\left(\int \sum_{i=1}^{n} h t_{j} x_{i}\right)=\sup _{h \in B_{L^{\infty}}}\left(\sum_{i=1}^{n} \alpha_{j}\left(h x_{i}\right)\right) .
\end{gathered}
$$

To see that (ii) $\Rightarrow$ (iv), fix $j \in \mathbb{N}$. If $J$ is a finite set of size $m$, write $S_{J}$ for the subspace generated by $\left\{t_{k}: k \in J\right\}$ and $Q_{J}$ for the corresponding projection map. For 
every finite set $J$ of size $m$ containing $j$, we have by Theorem 4.2 and considering the canonical isometry between $S_{J}$ and $\ell_{m}^{2}$ that

$$
\left\langle Q_{J} \circ T(x), t_{j}\right\rangle=\int h_{j}^{J} x t_{j}, d \mu, \quad x \in L^{2}[0,1] .
$$

Therefore, taking into account that $t_{k} \neq 0 \mu$-a.e. for every $k$, we obtain that $h_{j}^{J}=h_{j}^{J^{\prime}}$ for each pair of finite sets $J$ and $J^{\prime}$ containing $j$.

So, write now $h_{j}$ for the functions $h_{j}^{\{j\}}$. Fix a function $x \in L^{2}[0,1]$ and write $J_{N}$ for the sets $J_{N}=\{1, \ldots, N\}$. We have that

$$
T(x)(w)=\lim _{N} Q_{J_{N}} \circ T(x)=\lim _{N} \sum_{j=1}^{N}\left(\int h_{j} x t_{j} d \mu\right) t_{j}(w)=\sum_{j=1}^{\infty}\left(\int h_{j} x t_{j} d \mu\right) t_{j}(w) .
$$

This gives (iv) and finishes the proof, since a direct computation gives that (iv) $\Rightarrow$ (i).

Note that the corollary provides pointwise convergence of the sum, that is, for each fixed $x \in L^{2}[0,1]$. Since for each $x$ the corresponding trigonometric series is convergent, we have that the sequence of coefficients $\left(\int h_{j} x t_{j} d \mu\right)_{j=1}^{\infty}$ belongs to $\ell^{2}$, then by Carleson's Theorem the convergence is also $\mu$-a.e.

\section{References}

[1] J.M. Calabuig, O. Delgado and E.A. Sánchez Pérez, Generalized perfect spaces, Indag. Math. 19,3 (2008) 359-378.

[2] J.M. Calabuig, O. Delgado and E.A. Sánchez Pérez, Factorizing operators on Banach function spaces through spaces of multiplication operators, J. Math. Anal. Appl. 364, (2010) 88-103.

[3] O. Delgado and E. A. Sánchez Pérez, Summability properties for multiplication operators on Banach function spaces, Integr. Equ. Oper. Theory 66 (2010) 197-214.

[4] O. Delgado and E. A. Sánchez Pérez, Strong Factorizations between Couples of Operators on Banach Function Spaces J. Convex Anal. 20,3 (2013) 599-616.

[5] P. G. Dodds, C. B. Huijsmans and B. de Pagter, Characterizations of conditional expectation type operators, Pacific J. Math. 141,1 (1990) 55-77.

[6] J. Flores, F. L. Hernández and P. Tradacete, Domination problems for strictly singular operators and other related classes, Positivity 15,4 (2011) 595-616 (2011).

[7] D.H. Fremlin, Tensor products of Banach lattices, Math. Ann. 211, (1974) 87-106.

[8] G. Hu, Weighted norm inequalities for bilinear Fourier multiplier operators, Math. Ineq. Appl. 18,4 (2015), 1409-1425.

[9] P. Halmos and V. Sunder, Bounded integral operators on $L^{2}$ spaces, Springer, Berlin, 1978.

[10] L. Kantorovitch and B. Vulich. Sur la représentation des opérations linéaires, Compositio Mathematica 5 (1938) 119-165.

[11] P. Kolwicz, K. Leśnik and L. Maligranda, Pointwise multipliers of Calderón- Lozanovskii spaces, Math. Nachr. 286 (2013) 876-907.

[12] P. Kolwicz, K. Leśnik and L. Maligranda, Pointwise products of some Banach function spaces and factorization, J. Funct. Anal. 266,2 (2014) 616-659.

[13] W-C. Kuo, C.C.A. Labuschagne and B. A. Watson, Conditional expectations on Riesz spaces, J. Math. Anal. Appl. 303 (2005) 509-521. 
[14] J. Lindenstrauss and L. Tzafriri, Classical Banach Spaces II, Springer, Berlin, 1979.

[15] D. Maharam, The representation of abstract integrals, Trans. Am. Math. Soc. 75 (1953) 154-184.

[16] D. Maharam, On kernel representation of linear operators, Trans. Am. Math. Soc. 79 (1955) 229-255.

[17] L. Maligranda and L. E. Persson, Generalized duality of some Banach function spaces, Indag. Math. 51 (1989) 323-338.

[18] C.J. Neugebauer, Weighted norm inequalities for averaging operators of monotone functions, Publi. Mat. 35 (1991) 429-447.

[19] S. Okada, W. J. Ricker and E.A. Sánchez Pérez, Optimal Domain and Integral Extension of Operators acting in Function Spaces, Operator Theory: Adv. Appl., vol. 180, Birkhäuser, Basel, 2008.

[20] G. C. Rota, On the representation of averaging operators, Rend. Sem. Mat. Univ. Padova. 30 (1960) 52-64.

[21] E. A. Sánchez Pérez, Factorization theorems for multiplication operators on Banach function spaces, Integr. Equ. Oper. Theory 80,1 (2014) 117-135.

[22] A.R. Schep, Factorization of positive multilinear maps, Illinois J. Math. 28,4 (1984) 579-591.

[23] A.R. Schep, Products and factors of Banach function spaces, Positivity. 14,2 (2010) 301-319.

E. A. Sánchez Pérez

Instituto Universitario de Matemática Pura y Aplicada

Universitat Politècnica de València

Camino de Vera $\mathrm{s} / \mathrm{n}$

46022 Valencia

Spain

e-mail: easancpe@mat.upv.es 